\title{
Radiation Protection: Do We Need to Scan the Whole Lumbar Spine in Suspected Lumbar Spine Stenosis?
}

Tack ${ }^{1 *}$, Preziosi M', Cornil A ${ }^{1}$, Bohy $\mathbf{P}^{1}$, Katz $\mathbf{R}^{2}$, Van Muylem $\mathbf{A}^{3}$, Howarth $\mathbf{N}^{4}$ and Gevenois $\mathbf{P A}^{3}$

${ }^{1}$ Department of Radiology, Clinique de la Madeleine, Belgium

${ }^{2}$ Department of Radiology, CHU Ambroise Pare, Belgium ${ }^{3}$ Department of Pulmonology, Hopital Erasme, Belgium ${ }^{4}$ Department of Radiology, Cinique des Grangettes, Switzerland

*Corresponding author: Denis Tack, Department of Radiology, Clinique de la Madeleine, Epicura, Rue Maria Thomee 1, 6800 Ath, Belgium

Received: February 02, 2021; Accepted: March 19, 2021; Published: March 26, 2021

\begin{abstract}
Objectives: To test two hypotheses that the scan length could be reduced in patients younger than an age threshold below which lumbar stenosis in the two upper lumbar levels never occurs, and that an anteroposterior spinal canal diameter cut-off at the level of the L 3 pedicles could rule out a congenital stenosis at the L1 and/or L2 levels.
\end{abstract}

Methods: MR examinations of 55 healthy volunteers and 200 patients with suspected spinal canal stenosis were included. The anteroposterior diameter of the spinal canal was measured at each pedicle and each disk levels by two readers who also subjectively assessed the presence of stenosis.

Results: Degenerative spinal canal stenosis never occurs at the upper two lumbar disk levels in patients younger than 55 years. The anteroposterior diameter of the spinal canal diminished from L1 to L3 in both healthy volunteers and patients. An anteroposterior diameter of the spinal canal at the $L 3$ pedicles level $\geq 11 \mathrm{~mm}$ excluded a diameter $<10 \mathrm{~mm}$ at $\mathrm{L} 1$ and/or $\mathrm{L} 2$ pedicles levels.

Conclusion: A substantial reduction of the radiation dose from $\mathrm{CT}$ could be achieved by limiting the scan length from L3 to S1 in patients younger than 55 years provided that the anteroposterior diameter of the spinal canal is $>11 \mathrm{~mm}$ at the L3 pedicles level.

\section{Key points:}

- $\quad$ An anteroposterior diameter of the spinal canal at the level of the L3 pedicles $>11 \mathrm{~mm}$ rules out a congenital stenosis at the levels of $L 1$ and $L 2$.

- $\quad$ The CT acquisition length can be limited from L3 to S1 in patients younger than 55 years.

- $\quad$ Limiting the scan length from $L 3$ to $\mathrm{S} 1$ reduces the delivered radiation dose by 40 or $50 \%$ compared with scanning from L1 or Th12 to S1.

Keywords: Radiation protection; Computed tomography; X-ray lumbar vertebrae

\section{Abbreviations}

CT: Computed Tomography; ET: Echo Time; MR: Magnetic Resonance; NA: Not Applicable; ND: No Discordance; PACS: Archiving and Communicating System; ROC: Receiver Operating Characteristic; RT: Repetition Time; SD: Standard Deviation; ST: Slice Thickness; STIR: Short-T1 Inversion Recovery; 95\%CI: 95\% Confidence Interval; L1: First Lumbar Vertebral Body; L2: Second Lumbar Vertebral Body; L3: Third Lumbar Vertebral Body; L4: Fourth Lumbar Vertebral Body; L5: Fifth Lumbar Vertebral Body; L1-L2: First Lumbar Disk; L2-L3: Second Lumbar Disk; L3-L4: Third Lumbar Disk; L4-L5: Forth Lumbar Disk; L5-S1: Fifth Lumbar Disk; S1: First Sacrum Vertebral Body

\section{Introduction}

The lumbar canal normally narrows downward from L1 to S1 but its dimensions can be abnormally reduced. This phenomenon, so-called lumbar spinal stenosis, may be congenital or acquired, and is characterized by narrowing of the central spinal canal, lateral recess, and/or neural foramina at the level of lumbar vertebrae [1-4]. Acquired stenosis is often caused by osteoarthritis, disc degeneration, or ligament thickening, is related to ageing, and occurs most often in the lower lumbar spine [5]. Congenital stenosis occurs most often in the upper lumbar spine where it may cause neurogenic claudication [1-5]. The diagnosis is based on the patient's history, clinical signs, and cross-sectional imaging, either by Computed Tomography (CT) or Magnetic Resonance (MR), for confirming and localizing the spinal stenosis. Both techniques perform similarly with sensitivity ranging from 88 to $94 \%$ and specificity from 57 to $88 \%$ [5-9]. As it does not require ionizing radiation, MR should be preferred. Nevertheless, CT is frequently used as more accessible and less expensive than MR $[9,10]$, and like MR, CT can image the whole lumbar spine from T12 to $\mathrm{S} 1$ [10-12].

At CT, radiologists should optimize the radiation dose, particularly in young patients. Reducing the scan length contributes to this optimization [13]. Such a strategy could be applied in patients with suspected spinal stenosis, particularly in young patients in 
whom the stenosis may be congenital, provided that reduced scanned length does not result in misdiagnosis. Reducing this length from L2or even from L3-to S1 instead of scanning the whole lumbar spine from L1 to S1 will decrease the radiation dose by 20 to $40 \%$. Acquired stenosis-which can also occur in L1-L2 and/or L2-L3-is a degenerative disorder related to ageing. The scan length could be reduced in patients younger than an age threshold below which lumbar stenosis in the two upper lumbar levels never occurs. In addition, in order to not miss congenital stenosis in these patients, the scan length could be limited from L3 to S1 if the diameter of the lumbar canal in L3 reflects that at the level of L1 and L2. The aims of this study were therefore to investigate the relationships between $1^{\circ}$ ) patient's age and lumbar spine stenosis in order to find an age threshold, and $2^{\circ}$ ) canal dimensions in L1, L2, and L3, in both a group of patients referred for low back pain, sciatica, or suspected lumbar spine stenosis as well as in a group of normal asymptomatic volunteers.

\section{Materials and Methods}

\section{Patients}

Our local ethical committee approved this retrospective study and waived patient informed consent. As we do not routinely acquire CT scans of the whole lumbar spine (i.e., from T12 to S1), we based this study on MR. All patients referred for MR from July 2014 to June 2016 for low back pain, sciatica, or suspected lumbar spine stenosis, without a recent history of trauma, known neoplasm, or lumbar spine surgery and in whom a lumbar spine stenosis was either suspected or confirmed were retrieved from our Picture Archiving and Communicating System (PACS) (Telemis') and anonymized. In case of repeated examinations, only the first was retrieved. In addition, we included 55 asymptomatic healthy volunteers who were included in another study approved by the local ethical committee.

\section{Healthy volunteers}

The group of healthy volunteers included 55 subjects (18 men) aged from 21 to 63 -years-old (mean \pm SD); 36 years \pm 12 ) included in a previous (unpublished) study also approved by the local ethical committee. These subjects were recruited from the medical and paramedical staffs of our institution with the following inclusion criteria: age over 18; no prior consultation with a physician, physiotherapist, or osteopath for low back pain; no absence from work for low back pain; no previous history of spine trauma, spine infection, spine surgery, lumbar spine infiltration, neoplasia, or rheumatic disease, and no contra-indication to MR.

\section{MR technique}

MR was performed at 1,5 $\mathrm{T}$ in all patients (Aera; Siemens Healthineers). The protocol included sagittal T1 and T2-weighted images, axial T2-weighted images, focused on the levels of abnormalities, and T2-weighted myelographic images in patients with severe stenosis as evaluated by the radiologist in charge of the examination who was not involved in image reading for the present study. Parameters for axial T2-weighted images consisted of a repetition time (RT) of $3810 \mathrm{~ms}$, an Echo Time (ET) of $128 \mathrm{~ms}$ and a Slice Thickness (ST) of $3 \mathrm{~mm}$; for sagittal T2-weighted images, parameters consisted of $4480 \mathrm{~ms}, 120 \mathrm{~ms}$ and $4 \mathrm{~mm}$ respectively; for sagittal T1-weighted images, parameters consisted of $495 \mathrm{~ms}$, $8,6 \mathrm{~ms}$ and $4 \mathrm{~mm}$ respectively; for additional coronal myelographic sequences, parameters consisted of $5,45 \mathrm{~ms}, 2,73 \mathrm{~ms}$ et $1 \mathrm{~mm}$ respectively.

MR was performed at 1,5 $\mathrm{T}$ in all healthy volunteers (Intera, Philips Healthcare). The protocol included sagittal T1, T2 and axial STIR images. Only sagittal T1-weighted images sequences were used in this study and parameters consisted of a RT of $600 \mathrm{~ms}$, an ET of 13 $\mathrm{ms}$ and a slice thickness of $4 \mathrm{~mm}$.

All sequences used were anonymized and stored in the Picture Archiving and Communication System (PACS) by a radiology resident who was not involved in the image analysis.

\section{MR images reading}

MR images were viewed on a clinical workstation equipped with two color monitors with five megapixels resolution (Eizo $\mathrm{GmBH}$ ). Two radiologists (blinded for review) with respectively 15 and 30 years' experience in reading lumbar spine MR examinations were asked independently $1^{\circ}$ ) to measure with the caliper on the workstation the anteroposterior diameter of the lumbar spine canal of the dural sac-at the level of each pair of pedicles on the T1-weighted sagittal images and of each disk on the T2-weighted sagittal images (14), and $2^{\circ}$ ) on the basis of their visual assessment of T1 or T2-weighted images, to code the canal stenosis as present or absent at the level of each pair of pedicles and each disk.

In order to assess the intra-reader reproducibility, the 20 first MR examinations were read twice with a time interval of at least one month. In healthy volunteers, two other readers (blinded for review) with respectively 10 and three years of experience in reading lumbar spine MR examinations were asked to measure the anteroposterior diameter of the canal at the level of the pedicles of L1, L2, and L3.

\section{Statistical analysis}

According to consensus statements from the literature, we used a $10 \mathrm{~mm}$ anteroposterior diameter of the spinal canal as cut-off value to define the presence $(<10 \mathrm{~mm})$ or absence $(\geq 10 \mathrm{~mm})$ spinal canal stenosis [14-16]. The diameters of the spinal canal at the L1, L2, and L3 pedicles levels were compared in healthy volunteers by a linear mixed model (longitudinal model) including an order 1 auto-correlation structure, with level as fixed effect and a "by-subject" random effect. Inter- and intra-reader comparisons of anteroposterior diameter of the lumbar spine canal at the ten anatomical levels were made by t-paired tests. Inter- and intra-reader comparisons of frequencies of stenosis at the ten anatomical levels (number of stenosis at each level divided by the number of subjects) were made by a McNemar test. A Receiver Operating Characteristic (ROC) curve analysis was performed to assess the ability of L3 diameter to detect or exclude L1 or L2 diameter $\leq 10 \mathrm{~mm}$ [14-16]. The R software was used for statistical analyses [17]. A P-value lower than 0.05 was considered as statistically significant (two-tail).

\section{Results}

\section{Healthy volunteers}

The anteroposterior diameters of the spinal canal at the pedicles levels of L1 to L3 in the 55 healthy subjects are displayed in Figure 1.

\section{Patients}

Twelve, 37, and 61 patients were respectively excluded for history 


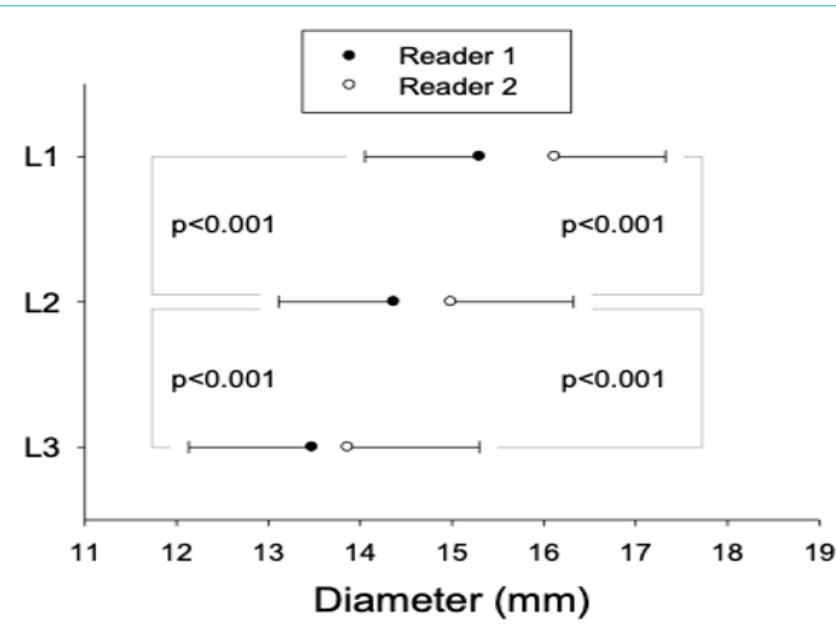

Figure 1: Anteroposterior diameter (mean \pm standard deviation) of the spinal canal measured by each reader at the levels of $L 1, L 2$, and $L 3$ pedicles in healthy volunteers.

Table 1: Mean \pm Standard Deviation of Anteroposterior Diameter of the Lumbar Spine Canal at the Ten Anatomical Levels in 200 Patients with Suspected Spinal Canal Stenosis and their Comparison between Readers.

\begin{tabular}{|c|c|c|c|c|}
\hline \multicolumn{2}{|c|}{ Level } & \multicolumn{2}{|c|}{ Diameter (mm) } & \multirow{2}{*}{ P-value $^{*}$} \\
\cline { 3 - 5 } & & Reader 1 & Reader 2 & \\
\hline \multirow{3}{*}{ Pedicles } & L1 & $14.4 \pm 1.5$ & $13.9 \pm 1.6$ & $<0.001$ \\
\cline { 2 - 5 } & L2 & $13.1 \pm 1.8$ & $13.0 \pm 1.8$ & 0.103 \\
\cline { 2 - 5 } & L3 & $11.7 \pm 2.0$ & $11.7 \pm 2.1$ & 0.561 \\
\cline { 2 - 5 } & L4 & $10.7 \pm 2.5$ & $10.9 \pm 2.5$ & 0.047 \\
\cline { 2 - 5 } & L5 & $10.1 \pm 2.9$ & $10.6 \pm 2.7$ & $<0.001$ \\
\hline \multirow{3}{*}{ Disk } & L1-L2 & $12.5 \pm 2.3$ & $12.2 \pm 2.5$ & $<0.001$ \\
\cline { 2 - 5 } & L2-L3 & $10.7 \pm 2.5$ & $10.4 \pm 2.7$ & $<0.001$ \\
\cline { 2 - 5 } & L3-L4 & $9.1 \pm 2.9$ & $9.1 \pm 3.0$ & 0.322 \\
\cline { 2 - 5 } & L4-L5 & $8.5 \pm 3.1$ & $8.6 \pm 3.2$ & 0.243 \\
\cline { 2 - 5 } & L5-S1 & $10.4 \pm 2.7$ & $10.6 \pm 2.7$ & $<0.001$ \\
\hline
\end{tabular}

Table 3: Frequency of Stenosis at Each Level: Inter-Reader Agreements in 200 Patients.

\begin{tabular}{|c|c|c|c|c|}
\hline \multicolumn{2}{|c|}{} & \multicolumn{2}{|c|}{ Frequency (\%) } & \\
\cline { 2 - 5 } & L1 & Reader 1 & Reader 2 & P-value $^{*}$ \\
\cline { 2 - 5 } & L2 & 0 & 0 & ND \\
\cline { 2 - 5 } & L3 & 0.5 & 0 & ND \\
\cline { 2 - 5 } & L4 & 10 & 1 & 0.564 \\
\cline { 2 - 5 } & L5 & 15.5 & 5.5 & 0.021 \\
\hline \multirow{3}{*}{ Dedicles Level } & L1-L2 & 1 & 1.5 & 0.564 \\
\cline { 2 - 5 } & L2-L3 & 7 & 8 & 0.317 \\
\cline { 2 - 5 } & L3-L4 & 24.5 & 26 & 0.491 \\
\cline { 2 - 5 } & L4-L5 & 40 & 38.5 & 0.513 \\
\cline { 2 - 5 } & L5-S1 & 8.5 & 7.5 & 0.564 \\
\hline
\end{tabular}

"Mc Nemar test; ND: No Discordance.

of recent trauma, known neoplasm, and lumbar spine surgery. Two hundred patients finally constituted our study group. There were 94 men and 106 women. Mean age ( \pm standard deviation, SD) was $56 \pm 16$. The indications for the MR examination were suspected lumbar spine stenosis (including claudication and bilateral inferior limbs paresthesia) in 132 patients (66\%), low back pain in 166 patients (83\%), right femoral-sciatic neuropathy in 50 patients $(25 \%)$, left femoral-sciatic neuropathy in 38 patients (19\%), and bilateral femoral-sciatic neuropathy in 40 patients (20\%).

The anteroposterior diameters of the lumbar spine canal measured by both readers in 200 patients with suspected spinal canal stenosis and their comparisons are summarized in Table 1. Results of the visual evaluation of the lumbar spine stenosis by both readers and their agreements are summarized in Table 2. The agreements within readers in the 20 first patients and the frequency of those with stenosis at each lumbar level are displayed in Table 2. The frequency of stenosis according to each level and the inter-reader agreements in 200 patients is displayed in Table 3 and displayed in Figure 2 according to patient's age. The diameters at the level of the L3 pedicles

Table 2: Visual Evaluation of Lumbar Spine Stenosis by Each Reader and their Agreements.

\begin{tabular}{|c|c|c|c|c|c|c|}
\hline & & \multirow{2}{*}{\multicolumn{2}{|c|}{ Number of Stenosis }} & \multicolumn{3}{|c|}{ Agreements (Kappa Coefficients) } \\
\hline & & & & \multirow{2}{*}{ Between Readers (200 patients) } & \multicolumn{2}{|c|}{ Within Readers (20 patients) } \\
\hline & & Reader 1 & Reader 2 & & Reader 1 & Reader 2 \\
\hline \multirow{5}{*}{ Pedicles Level } & L1 & 0 & 0 & NA & NA & NA \\
\hline & L2 & 0 & 0 & NA & NA & NA \\
\hline & L3 & 1 & 2 & -0.007 & NA & NA \\
\hline & L4 & 19 & 11 & 0.57 & 1 & 1 \\
\hline & L5 & 31 & 11 & 0.378 & 0.692 & 0.459 \\
\hline \multirow{5}{*}{ Disk Level } & L1-L2 & 2 & 3 & 0.392 & NA & NA \\
\hline & L2-L3 & 14 & 16 & 0.856 & 0 & 1 \\
\hline & L3-L4 & 49 & 52 & 0.748 & 0.138 & 0.138 \\
\hline & L4-L5 & 80 & 77 & 0.779 & 0.791 & 0.894 \\
\hline & L5-S1 & 17 & 15 & 0.593 & 0.318 & 1 \\
\hline
\end{tabular}

NA: Not Applicable. 
Table 4: ROC Curve Analysis: Assessment of L3 Diameter Ability to Predict or Exclude L1 or L2 diameter $\leq 10 \mathrm{~mm}$.

\begin{tabular}{|c|c|c|c|c|c|c|}
\hline & Prevalence $(\%)^{\star}$ & Area Under the Curve [IC95\%] & Sensitivity & Specificity & Positive predictive Value & Negative Predictive Value \\
\hline Reader 1 & 5 & $0.87[0.78-0.95]$ & $0.89^{* *}$ & $0.66^{* *}$ & $0.11^{* *}$ & $0.99^{* *}$ \\
\hline Reader 2 & 4 & $0.96[0.91-0.99]$ & $1.00^{* *}$ & $0.66^{* *}$ & $0.11^{* *}$ & $1.00^{* *}$ \\
\hline
\end{tabular}

: prevalence of $\mathrm{L} 1$ or $\mathrm{L} 2$ diameter $\leq 10 \mathrm{~mm}$.

: cutoff value of $\mathrm{L} 3$ diameter $=11 \mathrm{~mm}$.

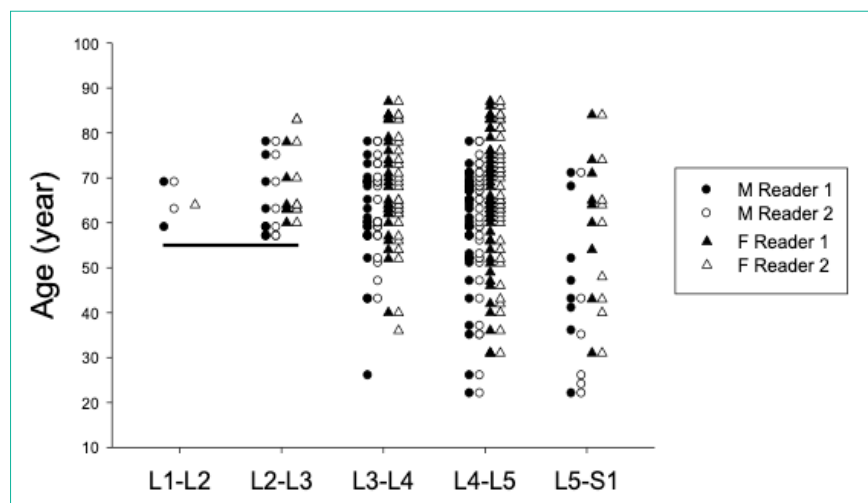

Figure 2: Number of spinal canal stenosis according to age and gender assessed by each reader. Horizontal line represents a 55 years age threshold for L1-L2 and L2-L3 lumbar disk levels.

associated with the anteroposterior diameters at the levels of both L1 and $\mathrm{L} 2$ pedicles level $\geq 10 \mathrm{~mm}$ or with one of these diameters $<10$ $\mathrm{mm}$ are displayed in Figure 3. As shown in this figure, there is a cut-off value of L3 diameter for discriminating normal $v s$. abnormal diameter superior or equal to $10 \mathrm{~mm} v s$. inferior to $10 \mathrm{~mm}$ in $\mathrm{L} 1$ or L2, considering this cut-off value as usually recommended and most frequently used by experts [14-16]. The cut-off values differed between readers and equal $10.1 \mathrm{~mm}$ for Reader 1 and $11.1 \mathrm{~mm}$ for Reader 2. We also calculated the area under the ROC curve that was 0.87 (95\% CI: 0.78-0.95) and 0.96 (95\% CI: 0.91-0.99), respectively for Reader 1 and 2 . The corresponding negative predictive value was 0.99 and 1.00, respectively for Readers 1 and 2 for an L3 diameter cutoff equal to $11 \mathrm{~mm}$. Sensitivity, specificity, and positive predictive values are reported in Table 4 for this cutoff value.

\section{Discussion}

This study shows the following: $1^{\circ}$ ) acquired spinal canal stenosis occurs between L3-L4 and L5-S1 in most of the patients (95\% in this study group); $2^{\circ}$ ) no acquired spinal canal stenosis at the levels L1L2 and L2-L3 occurs in patients younger than 55 years (44\% of our patients); $3^{\circ}$ ) as expected, the anteroposterior diameter of the spinal canal diminishes from $\mathrm{L} 1$ pedicles to L3 pedicles; $4^{\circ}$ ) a anteroposterior diameter $\geq 11 \mathrm{~mm}$ at the $\mathrm{L} 3$ pedicles level excludes a congenital spinal stenosis at levels of L1 and L2; and $5^{\circ}$ ) a diameter $\geq 10 \mathrm{~mm}$ at level of $\mathrm{L} 3$ pedicles has an almost perfect negative predictive value for excluding a spinal canal stenosis $(<10 \mathrm{~mm})$ at levels of L1 and L2. These findings deserve further discussion with regard to acquired and congenital spinal canal stenosis and have implications for radiation protection.

Acquired stenosis does not occur at L1 to L3 lumbar levels in patients younger than 55 years. These patients could therefore be scanned from L3 to S1 instead of L1 to S1, reducing the acquisition length and the subsequent dose-length product by approximately $40 \%$. Only patients older than 55 years would benefit from scanning

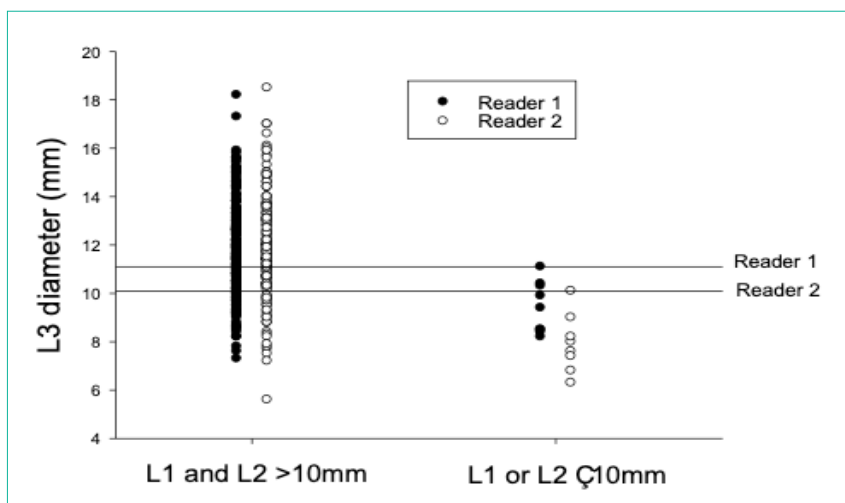

Figure 3: Anteroposterior diameter at the level of $L 3$ pedicles associated with diameters at levels $L 1$ and L2 pedicles $>10 \mathrm{~mm}$ versus $\leq 10 \mathrm{~mm}$. Horizontal lines indicate, for both readers, diameters at level of $L 3$ pedicle above which neither diameters at level L1 and L2 were $\leq 10 \mathrm{~mm}$.

their whole lumbar spine. The radiation-related risk is lower in these patients than in younger ones as this risk decreases with age [18]. However, reducing the acquisition length would be acceptable in patients younger than 55 years only if the anteroposterior diameter of their spinal canal measured at the L3 pedicles level could predict that at the L1 and L2 pedicles levels. This is confirmed by our findings with a $100 \%$ negative predictive value associated with the $11 \mathrm{~mm}$ cutoff value at the L3 pedicles level. In these patients, the anteroposterior diameter of the spinal canal at the L3 pedicles level could be measured before planning the actual acquisition length. This may be achieved by two means: first, a single reduced-dose axial scan at the level of the L3 pedicles before the helical acquisition with a dose of less than $1 \%$ of that delivered by the helical acquisition; or second, on scanners with large arrays of $16 \mathrm{~cm}$ length, the anteroposterior diameter at the L3 pedicles level could be measured on the first slice reconstructed from an acquisition range from $\mathrm{L} 3$ to $\mathrm{S} 1$. If this diameter is $<11 \mathrm{~mm}$, a second range could cover L1 to L3 and both image sets merged thereafter. As congenital spinal canal stenosis is rare, a vast majority of patients younger than 55 years would benefit from this $40 \%$ radiation dose reduction.

In terms of radiation protection and optimization, several strategies have been proposed to minimize the radiation dose delivered for imaging CT of the lumbar spine by CT. First, the tube current can be reduced by $35 \%$ compared with standard default protocols [9]. Second, iterative reconstructions allow further reductions through substantial noise reduction $[11,19]$. Third, acquisition parameters other than the tube current such as the automatic exposure control strength curve can be optimized, yielding a $10 \%$ possible additional reduction in all patients [20]. Our study shows that a potential $40 \%$ additional dose reduction is achievable in patients younger than 55 years by reducing the acquisition length from L3 pedicles to S1. This $40 \%$ dose reduction could even be up to $50 \%$ if the local routine acquisition length includes the whole lumbar spine from T12 pedicles 
as reported in surveys [21].

This study has some limitations. First, we assumed that MRI and CT were equivalent for assessing spinal canal stenosis and extrapolated findings from MRI to CT. Our assumption seems reasonable as both techniques perform well with very similar sensitivity (ranging from 88 to $94 \%$ ) and specificity (ranging from 57 to $88 \%$ ) [6-9]. Second, we included few patients with true congenital spinal stenosis. If its prevalence were higher, the observed negative predictive value of the anteroposterior diameter at the $\mathrm{L} 3$ pedicles level $\geq 10 \mathrm{~mm}$ would be reduced. The low proportion of congenital spinal stenosis however reflects our clinical practice and is likely to be observed elsewhere. Third, the disagreements between readers impact the negative predictive value of the diameter at the L3 pedicles level for ruling out a canal stenosis at the L1 or L2 levels. These discrepancies are well known and reflect the limitations of any lumbar spine imaging technique to predict the clinical outcome of patients with lumbar canal stenosis $[15,22]$. Finally, we limited our evaluation of the spinal canal to its anteroposterior diameter and did not consider the transverse surface of the dural sac. We were unable to do so as we did not obtain transverse scans with our MR system at all of the ten levels where we planned to measure the spinal canal. Nevertheless, for the diagnosis of lumbar spine stenosis, the use of the anteroposterior diameter measurement reaches a large consensus among experts [1416].

In conclusion, a substantial limitation of the radiation dose when scanning the lumbar spine could be implemented by limiting the scan range to L3-S1 in patients younger than 55 years provided that the anteroposterior diameter of the spinal canal could be measured $>11$ $\mathrm{mm}$ at the level of $\mathrm{L} 3$.

\section{References}

1. Anderson GBJ. Epidemiology of spinal disorders. Editor. In: Frymoyer JW The adult spine: principles and practice. New York, NY: Raven. 1997; 93-149.

2. Tubach F, Beaute J, Leclerc A. Natural history and prognostic indicators of sciatica. J Clin Epidemiol 2004; 57: 174-179.

3. Hillman M, Wright A, Rajaratnam G, Tennant A, Chamberlain MA. Prevalence of lowback pain in the community: implications for service provision in Bradford, UK. J Epidemiol Community Health. 1996; 50: 347-352.

4. Biering-Sorensen F, Thomsen C. Medical, social and occupational history as risk indicators for low-back trouble in a general population. Spine. 1986; 11: $720-725$.

5. Frymoyer JW. Back pain and sciatica. N Engl J Med. 1988; 318: 291-300.

6. Hudgins WR. Computer-aided diagnosis of lumbar disk herniation. Spine 1983; 8: 604-615.

7. Thornbury JR, Fryback DG, Turski PA, Javid MJ, McDonald JV, Beinlich BR, et al. Disk-caused nerve compression in patients with acute low-back pain: diagnosis with MR, CT myelography, and plain CT. Radiology. 1993; 186: 731-738.

8. Jackson RP, Cain JE, Jacobs RR, Cooper BR, McManus GE. The neuroradiographic diagnosis of lumbar herniated nucleus pulposus. II. A comparison of Computed Tomography (CT), myelography, CT-myelography, and magnetic resonance imaging. Spine. 1989; 14: 1362-1367.

9. Bohy P, De Maertelaer V, Roquigny A, Keyzer C, Tack D, Gevenois PA. Multidetector CT in patients suspected of having lumbar disk herniation: comparison of standard-dose and simulated low-dose techniques. Radiology. 2007; 244: 524-531.

10. Booz C, Noske J, Martin SS, Albrecht MH, Yel I, Lenga L, et al. Virtual noncalcium dual-energy CT: detection of lumbar disk herniation in comparison with standard grayscale CT. Radiology. 2019; 290: 446-455.

11. Gervaise A, Osemont B, Lecocq S, Noel A, Micard E, Felblinger J, et al. CT image quality improvement using adaptive iterative dose reduction with wide-volume acquisition on 320-detector CT. Eur Radiol. 2012; 22: 295-301.

12. Gervaise A, Louis M, Batch T, Loeuille D, Noel A, Guillemin F, et al. Dose reduction at CT of the lumbar spine using a 320-detector row scanner: initial results. J Radiol. 2010; 91: 779-785.

13. McNitt-Gray MF. AAPM/RSNA Physics Tutorial for Residents: Topics in CT. Radiation dose in CT. Radiographics. 2002; 22: 1541-1553.

14. Andreisek G, Deyo R, Jarvik JG, Porchet F, Winklhofer SFX, Steurer J. Consensus conference on core radiological parameters to describe lumbar stenosis-an initiative for structured reporting. Eur Radiol. 2014; 24: 32243232.

15. Steurer J, Roner S, Gnannt R, Hodler J. Quantitative radiologic criteria for the diagnosis of lumbar spinal stenosis: a systematic literature review. BMC Musculoskelet Disord. 2011; 12: 175.

16. Mamisch N, Brumann M, Hodler J, Held U, Brunner F, Steurer J. Radiologic criteria for the diagnosis of spinal stenosis: Results of a delphi survey. Radiology. 2012; 264: 174-179.

17. R Core Team R: a language and environment for statistical computing. 2021.

18. Hricak H, Brenner DJ, Adelstein SJ, Frush DP, Hall EJ, Howell RW, et al. Managing radiation use in medical imaging: a multifaceted challenge. Radiology. 2011; 258: 889-905.

19. Weinrich JM, Well L, Regier M, Behzadi C, Sehner S, Adam G, et al. MDCT in suspected lumbar spine fracture: comparison of standard and reduced dose settings using iterative reconstruction. Clin Radiol. 2018; 73: 675.e9-675.e15.

20. D'Hondt A, Cornil A, Bohy P, De Maertelaer V, Gevenois PA, Tack D. Tuning of automatic exposure control strength in lumbar spine CT. Br J Radiol. 2014; 87: 20130707.

21. Tack D, Jahnen A, Kohler S, Harpes N, De Maertelaer V, Back C, et al. Multidetector CT radiation dose optimisation in adults: short- and long-term effects of a clinical audit. Eur Radiol. 2014; 24: 169-175.

22. Andreisek G, Hodler J, Steurer J. Uncertainties in the diagnosis of lumbar spinal stenosis. Radiology. 2011; 261: 681-684. 